\title{
SUBSPACE BASED DETECTION IN MC-CDMA OVER DISPERSIVE CHANNEL
}

\author{
Chengyang Li and Sumit Roy \\ Dept. of EE, University of Washington, BOX 352500, Seattle, WA 98195-2500 \\ Email: cyli,roy@ee.washington.edu
}

\begin{abstract}
A new blind MMSE detection for Multi-Carrier CDMA (MC-CDMA [1]) over Intersymbol Interference (ISI) channels is proposed. The presence of virtual carriers [2] is exploited to give a subspace based blind channel estimator followed by a linear MMSE equalizer. The proposed detector is attractive for its higher spectral efficiency, which is achieved by the elimination of cyclic prefix and training sequences. The complexity of the full dimension subspace detection can be reduced at the cost of some performance degradation. Representative results are provided to show the performance-complexity trade-offs.
\end{abstract}

\section{INTRODUCTION}

Orthogonal Frequency-Division Multiplexing [3][4] (OFDM) is a well-known single-user modulation scheme that is bandwidth efficient. It also has several advantages, such as robustness to frequency selective fading, that render . it suitable for higher rate future standards. To equip the OFDM system with multiple access capability, various multi-carrier Code Division Multiple Accesș (CDMA) have been proposed in literature (see [5] for a good survey). These multi-carrier CDMA schemes follow the same spirit as OFDM - parallel transmission over large number of narrow band orthogonal carriers, yielding greater immunity against dispersive channels as compared to DS-CDMA for the same transmitted bandwidth.

In this work, we focus on the primary impairment for high data-rate systems - the presence of a dispersive channel, which is assumed to be essentially timeinvariant (or very slowly varying) that gives rise to ISI. The receiver design approach adopted is that of coherent multi-user detection which requires reliable channel estimation. Most previous works ([5]) suggest the use of cyclic prefix of length longer than the maximum delay spread of the channel to absorb the ISI. In this case, 0472

This work was supported in part by AFOSR Grant F49620-1- the dispersive channel effect reduces to a complex scaling factor in frequency on each sub channel. Thus a simple one-tap frequency-domain equalizer is applied to equalize the sub channel distortion. However, the price paid for using cyclic prefix is usually $10 \%-25 \%$ spectral efficiency loss [4]. Additionally, the required channel information is estimated usually by the insertion of training symbols, incurring a loss in channel utilization.

Our work is motivated by systems with high spectral efficiency which naturally leads to design approaches with no cylic prefix and blind channel estimation methods. In this paper, we demonstrate how the presence of 'virtual carriers' may be exploited for this purpose. The resulting blind detector architecture consists of a subspace based channel estimator [6] followed by a linear MMSE detector. Upon decomposition of the observation space into mutually orthogonal signal and noise subspaces, the channel information is estimated using the knowledge of the desired user's spreading code as in [7]; the channel estimate is then used to construct linear MMSE detector via direct off-line computation.

The rest of the paper is organized as follows: a baseband signal model for MC-CDMA system is introduced in Section 2. The subspace based channel estimator (both full and reduced dimension versions) and the linear MMSE detector are developed respectively in Section 3. Computer simulations are conducted in Section 4 to show the effectiveness of the proposed detector. And Section 5 concludes the paper.

\section{BASEBAND SIGNAL MODEL}

Consider an MC-CDMA system (see Fig. 1) with $Q$ subcarriers of which only $P$ are modulated by user's data symbols. The remaining $Q-P$ carriers are unmodulated to avoid transmit filtering and constitute virtual carriers. No cyclic prefix is used. $K$ users are multiplexed via their distinct $P$-length spreading sequences $\left(\mathbf{c}_{i}=\right.$ $\left[\begin{array}{llll}c_{i}(0) & c_{i}(1) & \ldots & c_{i}(P-1)\end{array}\right]^{T}$ for the $i$-th user). Denoting the transmitted information symbol for user $i$ in duration $n$ by $s_{i}(n)$, the signal after the IFFT operation 


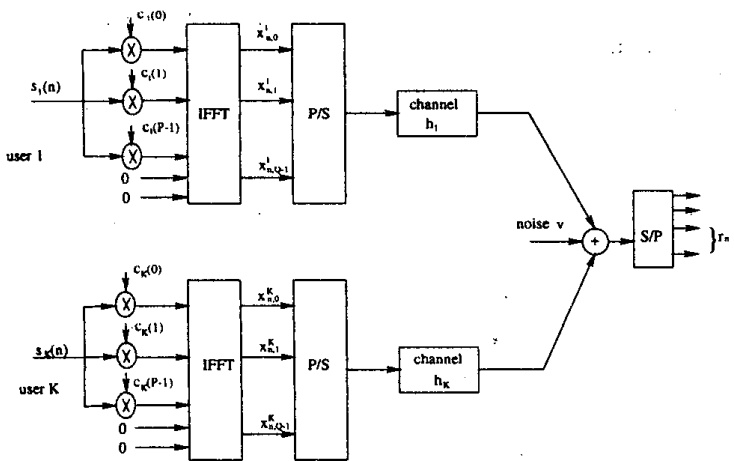

Figure 1: Baseband MC-CDMA system model

is

$$
\mathbf{x}_{i, n}=\left[x_{i, n}(0), \ldots, x_{i, n}(Q-1)\right]^{T}=\mathbf{W}_{P} \mathbf{c}_{i} s_{i}(n),
$$

where $\mathbf{W}_{P}$ is the $Q \times P$-dimensional partial IDFT matrix

$$
\mathbf{W}_{P}=\frac{1}{Q}\left[\begin{array}{cccc}
1 & 1 & \ldots & 1 \\
1 & W_{Q}^{-1} & \ldots & W_{Q}^{-(P-1)} \\
1 & W_{Q}^{-2} & \ldots & W_{Q}^{-2(P-1)} \\
\vdots & & & \vdots \\
1 & W_{Q}^{-(Q-1)} & \ldots & W_{Q}^{-(Q-1)(P-1)}
\end{array}\right]
$$

with $W_{Q}=e^{-j 2 \pi / Q}$. The dispersive channels are assumed to have length $L+1$, and the channel impulse response is denoted by $\mathbf{h}_{i}=\left[h_{i}(0), \ldots, h_{i}(L)\right]^{T}$ for user $i$. For convenience, the $i$-th user's transmit amplitude $\sqrt{E_{i}}$ is lumped into $\mathbf{h}_{i}$.

Upon transmission of $\mathbf{x}_{i, n}$ 's through the channel, the output is collected in a $Q$-vector

$$
\overline{\mathbf{r}}_{n}=\left[r_{n}(0), \ldots, r_{n}(Q-1)\right]^{T}=\sum_{i=1}^{K} \mathbf{x}_{i, n} * \mathbf{h}_{i}+\overline{\mathbf{v}}_{n}
$$

where $*$ respresents linear convolution, $\overline{\mathbf{v}}_{n}$ is an additive White Gaussian Noise (AWGN) vector consisting of independent, zero mean and variance $N_{0}$ components. The presence of the channel causes ISI from the past symbol in $\mathbf{r}_{n}$; thus we remove the first $L$ ISI affected samples and collect the remaining (ISI free) samples $\left(r_{n}(L)\right.$ to $\left.r_{n}(Q-1)\right)$ in duration $n$ into a vector

$$
\mathbf{r}_{n}=\sum_{i=1}^{K} \mathbf{H}_{i} \mathbf{W}_{P} \mathbf{c}_{i} s_{i}(n)+\mathbf{v}_{n}=\mathbf{A} \mathbf{s}_{n}+\mathbf{v}_{n} .
$$

with $\mathbf{s}_{n} \quad=\quad\left[s_{1}(n), \ldots, s_{K}(n)\right]^{T} \quad$ and $\mathbf{A}=\left[\mathbf{H}_{1} \mathbf{W}_{P} \dot{\mathbf{c}}_{1}, \ldots, \mathbf{H}_{K} \mathbf{W}_{P} \mathbf{c}_{K}\right]$.
Introducing the time-reversed version of $\mathbf{h}_{\mathbf{i}}$ as $\overline{\mathbf{h}_{\mathbf{i}}}=$ $\left[h_{i}(L), \ldots, h_{i}(0)\right]^{T}$, the $Q-L \times Q$-dimensional Toeplitz matrix $\mathbf{H}_{i}$ is given by

$$
\begin{aligned}
\mathbf{H}_{i} & =\operatorname{Toeplitz}\left(\overline{\mathbf{h}}_{i}\right) \\
& =\left[\begin{array}{cccccc}
h_{i}(L) & \ldots & h_{i}(0) & & & \\
& h_{i}(L) & \ldots & h_{i}(0) & & \\
& & \ddots & & \ddots & \\
& & & h_{i}(L) & \ldots & h_{i}(0)
\end{array}\right] .
\end{aligned}
$$

Further, define (borrowing notations from $\dot{M} A T L A B^{T M}$ ):

$$
\begin{aligned}
\overline{\mathbf{W}} & =\mathbf{W}_{P}(1: Q-L, 1: P), \\
\mathbf{W}_{L} & =\mathbf{W}_{P}(1: P, 1: L+1),
\end{aligned}
$$

where the ranges in parentheses represent the rows and columns of the corresponding matrix. Then, the scaled (by $Q$ ) IFFT output $\overline{\mathbf{g}}_{i}$, and $\mathbf{g}_{i}$ constructed from $\overline{\mathbf{g}}_{i}$ 's first $P$ elements are, respectively

$$
\begin{gathered}
\overline{\mathbf{g}}_{i}=\left[g_{i}(0), \ldots, g_{i}(Q-1)\right]^{T}=Q \mathbf{W}_{P} \overline{\mathbf{h}}_{i}, \\
\mathbf{g}_{i}=\left[g_{i}(0) \ldots, g_{i}(P-1)\right]^{T}=Q \mathbf{W}_{L} \overline{\mathbf{h}}_{i} .
\end{gathered}
$$

Now note that it can be shown the columns of $\mathbf{A}$ have the following structure:

$$
\mathbf{H}_{i} \mathbf{W}_{P} \mathbf{c}_{i}=Q \overline{\mathbf{W}} \operatorname{diag}\left(\mathbf{c}_{i}\right) \mathbf{W}_{L} \overline{\mathbf{h}}_{i}
$$

\section{BLIND MMSE DETECTION}

Without loss of generality, user 1 is denoted as the desired user and blind channel estimation of the channel vector $\mathbf{h}_{\mathbf{1}}$ via a subspace approach is outlined next. For notational convenience, the time index $n$ will be omitted in the following where there is no confusion.

\subsection{Subspace Based Blind Detection: Full Di- mension}

A subspace based channel estimation procedure can be readily derived from the signal model (3) provided that the necessary condition $Q-L \geq K$ for $\mathbf{A}$ to have full column rank is satisfied. The resulting algorithm exploits the special structure of $\mathbf{A}$ in (8) and is motivated by the approach in [7].

Assume each user's transmitted information symbols $s_{i}$ 's to be i.i.d. sequences with zero mean and unity variance. Consider the EigenValue Decomposition (EVD) on the correlation matrix of the observation vector $\mathbf{r}$ :

$\mathbf{R}_{\mathbf{r}}=E\left[\mathbf{r r}^{H}\right]=\left[\begin{array}{ll}\overline{\mathbf{U}}_{s} & \overline{\mathbf{U}}_{n}\end{array}\right]\left[\begin{array}{ll}\overline{\mathbf{\Lambda}}_{s} & \\ & \overline{\mathbf{\Lambda}}_{n}\end{array}\right]\left[\begin{array}{c}\overline{\mathbf{U}}_{s}^{H} \\ \overline{\mathbf{U}}_{n}^{H}\end{array}\right]$

where $\left[\overline{\mathbf{U}}_{s}, \overline{\mathbf{U}}_{n}\right]$ is an $Q-L \times Q-L$ unitary matrix. The $K$ columns of $\overline{\mathbf{U}}_{s}$ span the signal subspace, while 
$Q-L-K$ column vectors of $\overline{\mathbf{U}}_{n}$ span the orthogonal complement to the signal subspace, known as the noise subspace. $\bar{\Lambda}_{s}=\operatorname{diag}\left(\lambda_{1}, \lambda_{2}, \ldots, \lambda_{K}\right)$ is a diagonal matrix consisting of $K$ significant eigenvalues corresponding to the signal subspace, while $\bar{\Lambda}_{n}=N_{0} I_{Q-L-K}$ are the noise subspace eigenvalues. Since $\mathbf{H}_{1} \mathbf{W}_{P} \mathbf{c}_{1}$ lies in the subspace spanned by $\overline{\mathbf{U}}_{s}$, by the orthogonality property between signal subspace and noise subspace, it is orthogonal to $\overline{\mathrm{U}}_{n}$, namely by (8)

$$
\overline{\mathbf{U}}_{n}^{H} \mathbf{H}_{1} \mathbf{W}_{P^{-}} \mathbf{c}_{1}=\overline{\mathbf{U}}_{n}^{H} \overline{\mathbf{W}} \operatorname{diag}\left(\mathbf{c}_{1}\right) \mathbf{W}_{L} \overline{\mathbf{h}}_{1}=\mathbf{0}
$$

Eqn. (10) results in $Q-L-K$ equations for $L+1$ unknowns. Combining with the requirement that $Q-$ $L>P$ and the necessary condition for the identifiability (which needs $A$ to be full column rank, i.e., $Q-$ $L \geq K$ ), the number of users $K_{f}$ supported by the fulldimensioned system must satisfy

$$
K_{f}<\frac{2 Q-2 L-1}{2} \Rightarrow K_{f} \leq Q-L
$$

For the practical situation when the signal and noise subspaces are estimated from the sample covariance matrix $\hat{\mathbf{R}}_{\mathbf{r}}$, the above suggests the channel estimator

$$
\hat{\overline{\mathbf{h}}}_{1}=\arg \min _{\left\|\hat{\mathbf{h}}_{\mathbf{1}}\right\|=1}\left\|\hat{\overline{\mathbf{U}}}_{n}^{H} \overline{\mathbf{W}} \operatorname{diag}\left(\mathbf{c}_{1}\right) \mathbf{W}_{L} \overline{\mathbf{h}}_{\mathbf{1}}\right\|^{2}
$$

where $\hat{\overline{\mathbf{U}}}_{n}$ denotes the estimated noise subspace from $\hat{\mathbf{R}}_{\mathbf{r}}$. The solution to the above is well-known to be the eigenvector of $\mathbf{W}_{L}^{H} \operatorname{diag}\left(\mathbf{c}_{1}\right)^{H} \overrightarrow{\mathbf{W}}^{H} \hat{\mathbf{U}}_{n} \hat{\overline{\mathbf{U}}}_{n}^{H} \overline{\mathbf{W}} \operatorname{diag}\left(\mathbf{c}_{1}\right) \mathbf{W}_{L}$ corresponding to the minimum eigenvalue.

\subsection{Reduced-Dimension Blind Channel Estima- tion}

The complexity of the channel estimator presented above is $O\left((Q-L)^{3}\right)$ due to the EVD of the sample covariance matrix of the full-dimensioned received signal model in (3). In several scenarios, this complexity may be prohibitive for practical implementation - such as in downlink applications where the algorithm must be implemented in the transceiver on a mobile handset. Motivated by this, we now derive a reduced-dimension received signal model that leads to an $O(P)$ channel estimator with significant complexity reductions when $P<<$ $Q-L$.

Assuming that the necessary condition (8) is also sufficient in practice, the left pseudo-inverse matrix of $\overline{\mathbf{W}}$, i.e., $\tilde{\mathbf{W}}=\left(\overline{\mathbf{W}}^{H} \overline{\mathbf{W}}\right)^{-1} \overline{\mathbf{W}}^{H}$ can be applied to (3) yielding

$$
\dot{\overline{\mathbf{y}}}=\tilde{\mathbf{W}} \mathbf{r}=\left[\begin{array}{llll}
\mathbf{f}_{1} & \mathbf{f}_{2} & \ldots & \mathbf{f}_{K}
\end{array}\right] \mathbf{s}+\mathbf{z}
$$

where from (8), $\mathbf{f}_{i}=Q \operatorname{diag}\left(\mathbf{c}_{i}\right) \mathbf{W}_{L} \overline{\mathbf{h}}_{i}$. Note that the additive noise component $\mathbf{z}$ is now colored with covariance given by $\mathbf{R}_{\mathbf{z}}=N_{0}\left(\overline{\mathbf{W}}^{H} \hat{\mathbf{W}}\right)^{-1}$. Since $\left(\overline{\mathbf{W}}^{H} \overline{\mathbf{W}}\right)^{-1}$ is a positive define Hermitian matrix, it admits a Cholesky factorization $\left(\overline{\mathbf{W}}^{H} \overline{\mathbf{W}}\right)^{-1}=\mathbf{B B}^{H}$. An equivalent received signal model in additive white noise results by applying $\mathbf{B}^{-1}$ to the signal vector $\overline{\mathbf{y}}$ in (13), i.e.,

$$
\mathbf{y}=\mathbf{B}^{-1} \overline{\mathbf{y}}=\mathbf{B}^{-1} \tilde{\mathbf{W}} \mathbf{r}
$$

A low complexity channel estimator based on (14) can be obtained after the raw observation vector $\mathbf{r}$ is prefiltered via $\mathbf{D}=\mathbf{B}^{-1} \tilde{\mathbf{W}}$ to produce the (reduced) $P$ dimension observation vector $\mathbf{y}$. The pre-filtexing results in no loss of information for purposes of detection, i.e., the observation $\mathbf{y}$ is a sufficient statistic.

Similar to the full dimension estimator, the covariance matrix of the observation vector $\mathbf{y}$ can be decomposed via EVD as

$\mathbf{R}_{\mathbf{y}}=E\left[\mathbf{y} \mathbf{y}^{H}\right]=\left[\begin{array}{ll}\mathbf{U}_{s} & \mathbf{U}_{n}\end{array}\right]\left[\begin{array}{ll}\boldsymbol{\Lambda}_{s} & \\ & \boldsymbol{\Lambda}_{n}\end{array}\right]\left[\begin{array}{l}\mathbf{U}_{s}^{H} \\ \mathbf{U}_{n}^{H}\end{array}\right]$

where the signal subspace is spanned by the $K$ columns of $\mathrm{U}_{s}$, while the noise subspace is now spanned by $P-$ $K$ column vectors of $\mathbf{U}_{n} ; \boldsymbol{\Lambda}_{s}=\operatorname{diag}\left(\lambda_{1}, \lambda_{2}, \ldots, \lambda_{K}\right)$ and $\Lambda_{n}=N_{0} I_{P-K}$ are diagonal matrices constituted by eigenvalues corresponding to the signal subspace and noise subspace respectively.

When only estimated noise and signal subspaces $\left(\hat{\mathbf{U}}_{n}\right.$ and $\hat{U}_{s}$ ) are available from EVD of sample covariance matrix $\hat{\mathbf{R}}_{\mathbf{y}}$, the desired user's channel vector $\overline{\mathbf{h}}_{\mathbf{1}}$ is determined by the Least-Square (LS) solution of

$$
\hat{\mathbf{U}}_{n}^{H} \mathbf{B}^{-1} \operatorname{diag}\left(\mathbf{c}_{1}\right) \cdot \mathbf{W}_{L} \overline{\mathbf{h}}_{1} \approx 0
$$

Eqn. (16) results in $P-K$ equations for $L+1$ unknowns. Combining with the requirement that $Q-$ $L>P$ and the necessary condition for the identifiability (which needs $\mathbf{B}^{-1} \tilde{\mathbf{W}} \mathbf{A}$ to be full column rank, i.e., $P>K)$, the number of users in the reduced dimension system must satisfy

$$
K_{r}<\frac{Q+P-2 L-1}{2} \Rightarrow K_{r} \leq \frac{Q+P}{2}-L
$$

Comparing with (11), it is evident that the full-dimension system can support more users since $Q>P$.

\subsection{MMSE Detector Computation}

Once $\hat{\mathbf{h}}_{\mathbf{1}}$ is available, the optimal coefficient vector $\mathbf{w}_{\text {opt }}$ for a linear MMSE equalizer for the desired user is determined by solving $\mathbf{w}_{\text {opt }}=\arg \min _{\mathbf{w}} E\left[\left\|s_{1}-\hat{s_{1}}\right\|^{2}\right]$, where $\hat{s_{1}}$ is the estimate of the transmitted symbol $s_{1}$

$$
\begin{aligned}
& \hat{s_{1}}=\mathbf{w}^{H} \mathbf{r} \text { (full dimension detector) } \\
& \hat{s_{1}}=\mathbf{w}^{H} \mathbf{y} \text { (reduced dimension detector) }
\end{aligned}
$$


A. Full Dimension MMSE Detector: The optimal equalizer weight vector for the full dimension detector $\mathbf{w}_{\mathbf{o p t}, \mathbf{r}}$ which minimizes the MSE between $s_{1}$ and its estimate $\hat{s_{1}}$ is

$$
\mathbf{W}_{\text {opt }, \mathbf{r}}=\overline{\mathbf{U}}_{s} \bar{\Lambda}_{s}^{-1} \overline{\mathbf{U}}_{s}^{H} \overline{\mathbf{W}} \operatorname{diag}\left(\mathbf{c}_{1}\right) \mathbf{W}_{L} \hat{\mathbf{h}}_{1}
$$

In practice, $\overline{\mathrm{U}}_{s}$ and $\bar{\Lambda}_{s}$ are replaced by their estimated version $\hat{\bar{U}}_{s}$ and B. Reduced Dimension MMSE Detector: Similarly, the optimal equalizer weight vector $\mathbf{w}_{\text {opt,y }}$ for the reduced dimension detector is

$$
\mathbf{w}_{o p t, \mathbf{y}}=\hat{\mathbf{U}}_{s} \hat{\Lambda}_{s}^{-1} \hat{\mathbf{U}}_{s}^{H} \mathrm{~B}^{-1} \operatorname{diag}\left(\mathbf{c}_{1}\right) \mathbf{W}_{L} \hat{\mathbf{h}}_{1}
$$

It is worth remarking that the optimum MMSE detector lies in signal subspace, as is evident from (20) or (21).

\section{SIMULATION RESULTS}

Monte Carlo simulations were conducted to assess the effectiveness of the proposed blind detectors. The channels were generated using the method of [8] that models the channel as a finite length, tapped delay line with an input Doppler frequency parameter that controls the rate of time variation. We set the Doppler frequency to zero to get time-invariant ISI channels.

We consider an MC-CDMA system with no cyclic prefix with $Q=16$. Number of data subcarriers $P$ is set to 10 unless otherwise noted; the channel length $L+1$ is 3 or 4 . $P$-length random binary codes are applied to BPSK data symbols at the transmitter during each run; the codes and channel realizations are independently selected for each run of the simulation, thereby resulting in averaged performance over the choice of such codes and channels. The $i$-th user's transmit SNR is defined as $S N R_{i} \stackrel{\text { def }}{=} 10 \log _{10} \frac{E_{i}}{N_{0}}$. Both single user and multiple users cases are simulated. The sample average of the autocorrelation matrix $\mathbf{R}_{\mathbf{r}}$ (or $\mathbf{R}_{\mathbf{y}}$ ) is obtained by using 1000 samples of $\mathbf{r}_{n}$ (or $\mathbf{y}_{n}$ ). All results reported are obtained by averaging over 300 independent Monte Carlo runs.

Performance of the channel estimator: Performance of the proposed channel estimator is evaluated in terms of normalized Root Mean-Square Error (RMSE). Fig. 2 illustrates the RMSE comparison between the full and reduced dimension channel estimator when two active users are present. The channel length $L+1$ is 3 and 4 . The comparison clearly shows that reduced dimension estimator suffers higher RMSE than the full dimension estimator, with their performance converging at suffciently high SNR ( $>20 \mathrm{~dB}$ ). Further, increasing the channel order by 1 incurs a maximum $3 \mathrm{~dB}$ penalty for fixed $K$.

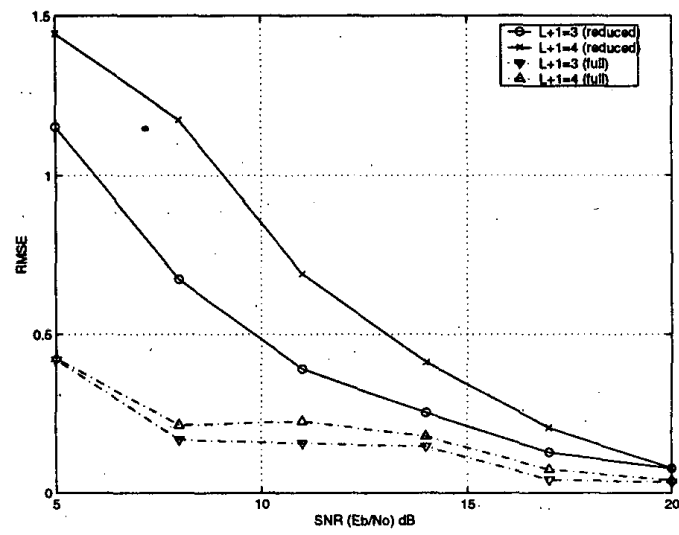

Figure 2: RMSE comparison between full and reduced dimension estimator

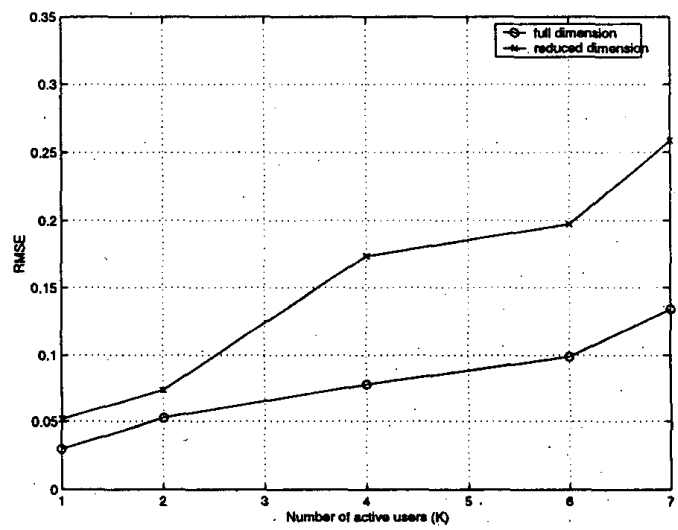

Figure 3: RMSE Vs. number of active users $K$

Next, the impact of increasing the number of users is shown in Fig. 3; where the channel length is set to 3 and the SNR is fixed at $15 \mathrm{~dB}$. The lower rate of RMSE increasing for the full dimension estimator reflects its better noise tolerance property over the dimension-reduced estimator.

The effect of difference selection of $P$ is illustrated in Fig. 4. The channel length $L+1$, the active users $K$, and the input SNR are fixed as 3,2 and $20 \mathrm{~dB}$ respectively. As expected, larger $P$ (which is the processing gain in traditional CDMA) gives better channel estimation.

BER Performance of the MMSE Detector: Bit Error Rate (BER) is plotted as an indicator of the MMSE detector. In Fig. 5, the BER curves for both the full and reduced dimension detector are shown together for com- 


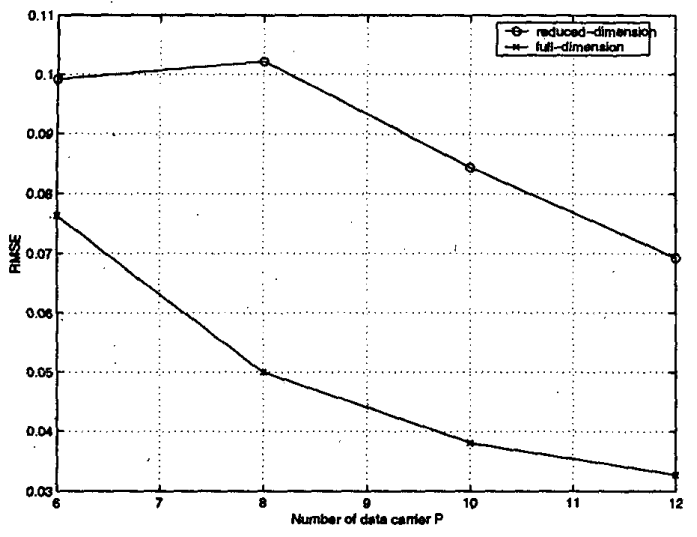

Figure 4: RMSE vs. P

parison, where the channel length $L+1=3$ and all users ( $K=1,2$ or 4$)$ have same transmit power. The reduced

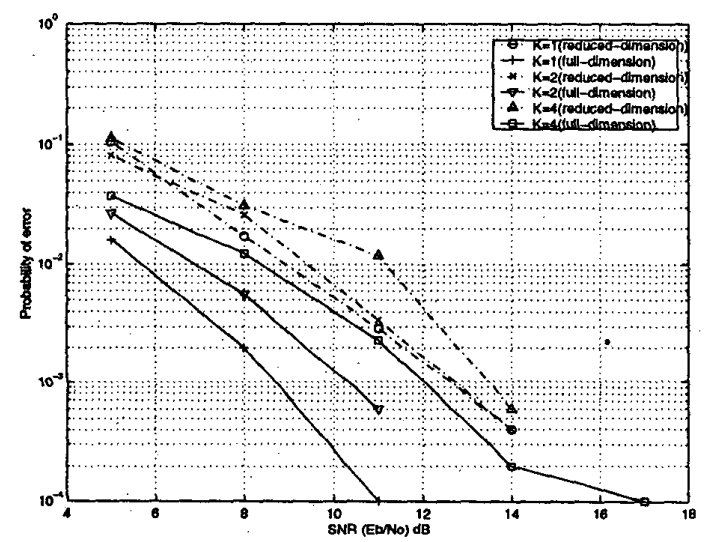

Figure 5: Bit Error Rate for the full and reduced dimension detector $(L+1=3)$

complexity detector results in higher BER than the fulldimension detector due to it's poorer channel estimation accuracy. However, at moderate SNR (14dB), the reduced complexity detector achieves (acceptable) BER below $10^{-3}$. Note that the SNR penalty for the full vs. reduced-dimensioned detector decreases with increasing number of users - it is less than $4 \mathrm{~dB}$ for single user, about $2.2 \mathrm{~dB}$ for $K=2$ and less than $2 \mathrm{~dB}$ when $K=4$.

\section{CONCLUSION}

A new blind channel estimator for MC-CDMA systems that exploit the presence of virtual carriers was presented. The proposed detector architecture allows prefiltering for dimensionality reduction in interests of reduced complexity at the expense of some performance degradation. Simulation results cataloged detector performance as a function of the relevant system parameters such as channel length and number of users. Future extension of this work will incorporate adaptivity into the detector to capture the time-varying (fading multipath) nature of typical wireless channel.

\section{REFERENCES}

[1] N.Yee, J-P.Linnartz and G.Fettweis, "Multicarrier CDMA in indoor wireless radio networks", in Proc. of IEEE PIMRC93, pp.109-13, 1993.

[2] H.Sari, G.Karam, and I.Jeanclaude, "Transmission techniques for digital terrestrial TV broadcasting", IEEE Commun. Mag., vol. 33, no.2, pp. 100-109, Feb. 1995.

[3] J.A.C. Bingham, "Multicarrier modulations for data transmission: An idea whose time has come", IEEE Commun. Mag., vol. 28, no.5, pp. 5-14, May 1990.

[4] W.Y. Zou and Y. Wu, "COFDM: An overview", IEEE Trans. Broadcasting, vol. 41, pp. 1-8, Mar. 1995.

[5] S.Hara and R.Prasad, "Overview of Multicarrier CDMA", IEEE Commun. Mag., vol. 35, No. 12, pp.126-133, Dec. 1997.

[6] E. Moulines, P. Duhamel, J. Cardoso, and S. Mayrargue, "Subspace methods for the blind identification of multichannel FIR filters", IEEE Trans. on Sig. Proc., vol. 43, no. 2, pp.516-525, Feb. 1995.

[7] $\mathrm{H}$. Liu and G. Xu, "A subspace method for signature waveform estimation in synchronous CDMA system", IEEE Trans. Commun., vol. 44 , no.10, pp.1346-1354, Oct. 1996.

[8] P.Hoeher, "A statistical discrete-time model for the WSSUS multipath channel", IEEE Trans. Veh. Tech., vol. 41, no.4, pp.461-468, Nov. 1992. 